\title{
Correction to: A Minimal Closed-Form Solution for Multi-perspective Pose Estimation using Points and Lines
}

Pedro Miraldo, Tiago Dias, and Srikumar Ramalingam

\section{Correction to:}

Chapter "A Minimal Closed-Form Solution

for Multi-perspective Pose Estimation using Points and Lines"

in: V. Ferrari et al. (Eds.): Computer Vision - ECCV 2018,

LNCS 11220, https://doi.org/10.1007/978-3-030-01270-0_29

The original version of this chapter unfortunately contained a mistake. The presentation of Eq. 5 was incorrect. The original chapter has been corrected. 